\title{
Permeability of blood-retinal barriers in urethane-induced rat retinopathy: a fluorescein angiographic, vitreous fluorophotometric, and fluorescence microscopic study
}

\author{
ERNA E. KRITZINGER ${ }^{1}$ AND ROY W. BELLHORN ${ }^{2}$ \\ From the 'Birmingham and Midland Eye Hospital, Birmingham, England and the \\ ${ }^{2}$ Department of Ophthalmology, Montefiore Hospital and Medical Center/Albert Einstein College \\ of Medicine, Bronx, NY USA
}

SUMMARY Urethane-induced rat retinopathy, characterised by permeability abnormalities of the blood-retinal barriers (BRB), was studied during the developmental phases by fluorescein fundus angiography (FFA), vitreous fluorophotometry (VF), and fluorescence microscopy (FM). A distinction based on VF values could be made at the $p<0.02$ confidence level between the retinopathic rats as a group and the control rats. Fluorescence microscopy provided a basis, however, for subdividing the test group into those rats with evidence of intraretinal leakage of $\mathrm{NaFl}$ and those without. Statistical analysis of the VF values of the control (A), nonleaky retinopathic (B), and leaky retinopathic (C) rats showed no significance between groups A and B, but highly significant differences between groups $A$ and $C(p<0.001)$ and between groups $B$ and $C(p<0.01)$. Fluorescence microscopy also showed that leakage of $\mathrm{NaFl}$ from retinal vessels occurred only after the retinopathy has progressed to the point where retinal vessels had become incorporated into the pigment epithelium. We conclude from this fluorescent marker clinicopathological study that breakdown of the blood-retinal barriers is a result of an interaction between the retinal pigment epithelium (RPE) and the retinal vessels after the vessels become incorporated into the RPE.

The severity of vascular retinopathies has been assessed by ophthalmoscopy and fundus slit-lamp biomicroscopy for some decades. Since decribed by Novotny and Alvis in $1961,{ }^{1}$ fundus fluorescein angiography has been utilised to define in greater topographical detail the permeability abnormalities of the blood-retinal barrier (BRB) associated with vascular retinopathies. Subsequently, in 1975, vitreous fluorophotometry was reported by CunhaVaz et al. ${ }^{2}$ as a more sensitive method of detecting and quantitating these abnormalities. The interpretation of clinical data obtained with these techniques is not always clear in relation to the tissue(s) involved in the permeability disturbance; this is especially true of vitreous fluorophotometry. In experimental ophthalmic research, however, these tissues can be

Correspondence to Miss Erna E. Kritzinger, FRCS, Birmingham and Midland Eye Hospital, Church Street, Birmingham B3 2NS, England. identified by use of the same marker, namely, sodium fluorescein, via the techniques of freeze-dry tissue preparation and fluorescence microscopy..$^{3-5}$ Several studies of experimental rat retinopathies have used fluorescein angiography ${ }^{67}$ and vitreous fluorophotometry $^{8-11}$ to demonstrate abnormalities of the BRB, but so far fluorescence microscopic localisation of fluorescein has been reported in only one instance. ${ }^{4}$

The present study concerns a clinicopathological correlation of the early phases of urethane-induced rat retinopathy ${ }^{6}$ by means of the complimentary techniques of fundus fluorescein angiography (FFA), vitreous fluorophotometry (VF), and freeze-dry tissue fluoresence microscopy (FM).

\section{Materials and methods}

An experimental vascular retinopathy was induced in pigmented rats of the Long-Evans strain by sub- 
cutaneous injection of urethane. ${ }^{6}$ Newborn litters were so divided that 12 rats served as test and 7 rats as control animals. The test animals were injected with 1 $\mathrm{mg}$ urethane per gram bodyweight on their day of birth and then at weekly intervals for 8 weeks. Urethane was dissolved in distilled water, $100 \mathrm{mg}$ per $\mathrm{ml}$. The 7 control animals received injections containing only distilled water.

Evaluation of the control and test animals was carried out at biweekly intervals from week 10 to week 16 by the techniques described below.

Fundus ophthalmoscopy. After instillation of $1 \%$ atropine sulphate and $10 \%$ phenylephrine hydrochloride to dilate the pupils the fundus was examined by indirect ophthalmoscopy. Colour photographs were taken with a Zeiss fundus camera using Kodak Ektachrome ASA 64 film.

Fundus fluorescein angiography (FFA). FFA was accomplished via the femoral vein on anaesthetised rats as described by Bellhorn et al. ${ }^{12}$ The dosage of sodium fluorescein used was $33 \mathrm{mg}$ per $\mathrm{kg}$ bodyweight and the duration of the angiographic sequence was 30 minutes.

Vitreous fluorophotometry (VF). Leakage of sodium fluorescein into the vitreous was measured one-half hour after FFA (1 hour after injection) according to the technique described by Cunha-Vaz et $a l^{2}{ }^{2}$ The concentration of sodium fluorescein was determined at midvitreous level, background fluorescence having been recorded before injection. The VF was performed by a single observer (E.E.K.), who was unaware of the treatment status of the animal. The equipment consisted of a slit-lamp (Haag-Streit model 900) with a $450 \mu \mathrm{m}$ diameter photometric probe (Gamma Scientific model 700-1044 ) inserted into the right ocular. A slit beam, size $0.5 \times 3 \mathrm{~mm}$, was used with the angle between the arm and the ocular of the slit-lamp set at 20 degrees. The photometric tube was connected to a photomultiplier, and signals were read with a digital radiometer (Gamma Scientific model DR-IA). Spectrotech SE40 excitation and Micro-optics CWL 570 barrier filters were used. The equipment was calibrated with freshly prepared serial solutions of sodium fluorescein in normal saline to give a linear response over the range $10^{-5}$ to $10^{-10} \mathrm{~g} / \mathrm{ml}$. The sensitivity of the system was adjusted to yield a signal intensity at least twice as large as that of background noise with an input voltage of $960 \mathrm{mV}$. Pre- and postinjection plasma reading times corresponded with those of VF. Results were expressed as vitreous:plasma ratio (V:P), after correction for fundus and plasma background fluorescence. They were compared by the Student's $t$ test.

Light and fluorescence microscopy (FM). After the above mentioned procedures the rats were allowed to
Table 1 Summary of results

\begin{tabular}{|c|c|c|c|c|}
\hline $\begin{array}{l}\text { Animal } \\
\text { Group }\end{array}$ & $\begin{array}{l}\text { FFA } \\
\text { leakage } \\
\text { suspected }\end{array}$ & $\begin{array}{l}V F \\
V: P(\text { mean })\end{array}$ & $\begin{array}{l}F M \\
\text { vessels } \\
\text { in } R P E\end{array}$ & $\begin{array}{l}\text { FM } \\
\text { leakage }\end{array}$ \\
\hline $\begin{array}{l}\text { Group A } \\
(n=7)\end{array}$ & - & $\begin{array}{l}0.016 \\
S D=0.009 \\
S E=0.004\end{array}$ & - & - \\
\hline $\begin{array}{l}\text { Group B } \\
(n=5)\end{array}$ & \pm & $\begin{array}{l}0 \cdot 019 \\
S D=0.010 \\
S E=0.005\end{array}$ & \pm & - \\
\hline $\begin{array}{l}\text { Group C } \\
(n=7)\end{array}$ & + & $\begin{array}{l}0.036 \\
S D=0.005 \\
S E=0.002\end{array}$ & + & + \\
\hline $\begin{array}{l}\text { Group B \& C } \\
(n=12)\end{array}$ & & $\begin{array}{l}0.029 \\
S D=0.011 \\
S E=0.003\end{array}$ & & \\
\hline
\end{tabular}

$\mathrm{RPE}=$ retinal pigment epithelium; $\pm=$ some rats positive, others negative.

survive for at least 7 days before enucleation of the eyes. They were again anaesthetised and injected intravenously with a $0.5 \%$ solution of sodium fluorescein, $0.1 \mathrm{ml}$ per $200 \mathrm{~g}$ bodyweight, which was allowed to circulate for 5 minutes. After enucleation of the eyes the rats were killed with an overdose of sodium pentobarbital. The eye on which the VF had been performed was prepared for fluorescence microscopy, as previously described. ${ }^{5}$ The tissue sections were viewed with a Zeiss Photomicroscope 11 equipped with exciter (BG12) and barrier (Zeiss 47) filters for epi-illumination. Photographs were taken with Kodak Ektachrome ASA 400 film. The opposite eye was prepared for light microscopy with the plastic embedding technique. ${ }^{6}$ The embedded tissues were sectioned at $2 \mu \mathrm{m}$ and stained with toluidine blue.

\section{Results}

Ophthalmoscopy showed that the test animals had developed the typical features of urethane-induced retinopathy, ${ }^{6}$ that is, interspersed areas of hypo- and hyperpigmentation were visible, mainly in the posterior fundus, and the retinal vessels were irregular in outline. The fundi of the control rats were normal. Angiography of the test animals (Table 1) showed areas of capillary dropout and, in some, diffuse patterns of dye suggestive of leakage of fluorescein into the retina (Fig. 1A). In contrast the angiograms of the control animals showed full capillary beds and no leakage of dye (Fig. 1B).

Examination of histological sections confirmed that the test animals had developed the retinopathy as described. ${ }^{6}$ Loss of the outer retinal layers was noted in all the rats; in those rats with later phase retinopathy, extension of retinal vessels into the 


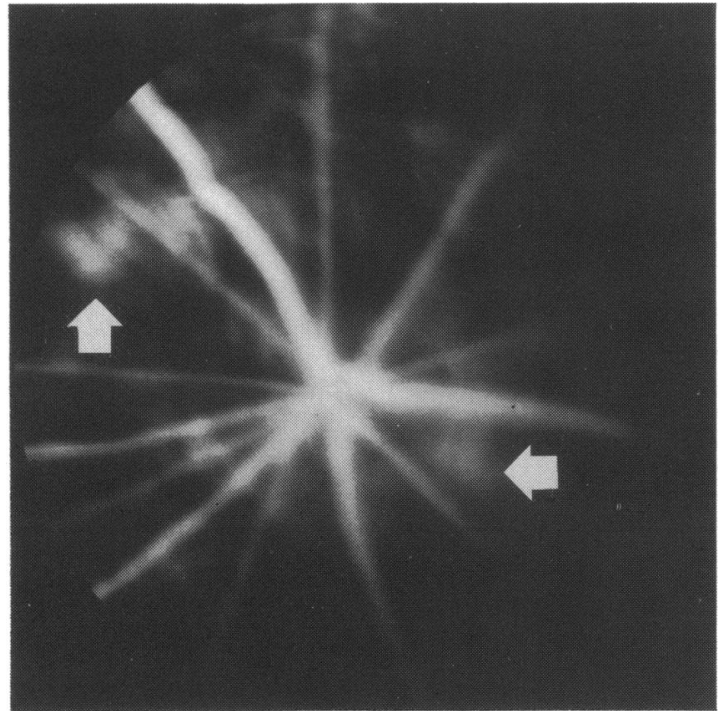

Fig. 1a Fluorescein angiogram of urethane retinopathic rat showing poor capillary perfusion and diffuse patches of fluorescence suggestive of deep retinal leakage (arrows).

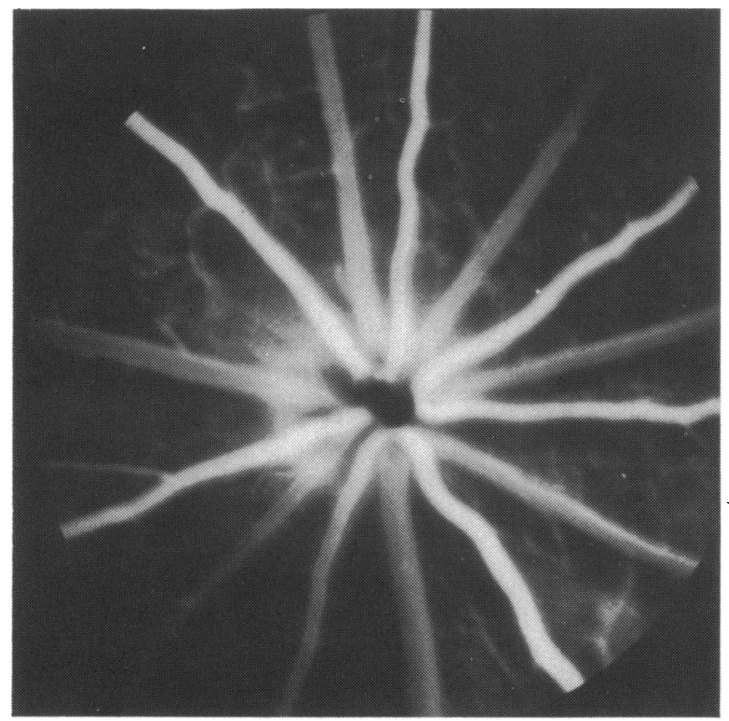

Fig. 1b Fluorescein angiogram of control rat showing good filling of capillary beds.

underlying retinal pigment epithelium (Fig. 2A) was present (Table 1). The control animals showed normal retinal stratification with absence of vessels in the retinal pigment epithelium (Fig. 2B).

In general the duration of the retinopathy was correlated with the severity; however, this relationship was not entirely consistent in that some rats 10-12 weeks old had a more severe retinopathy than some

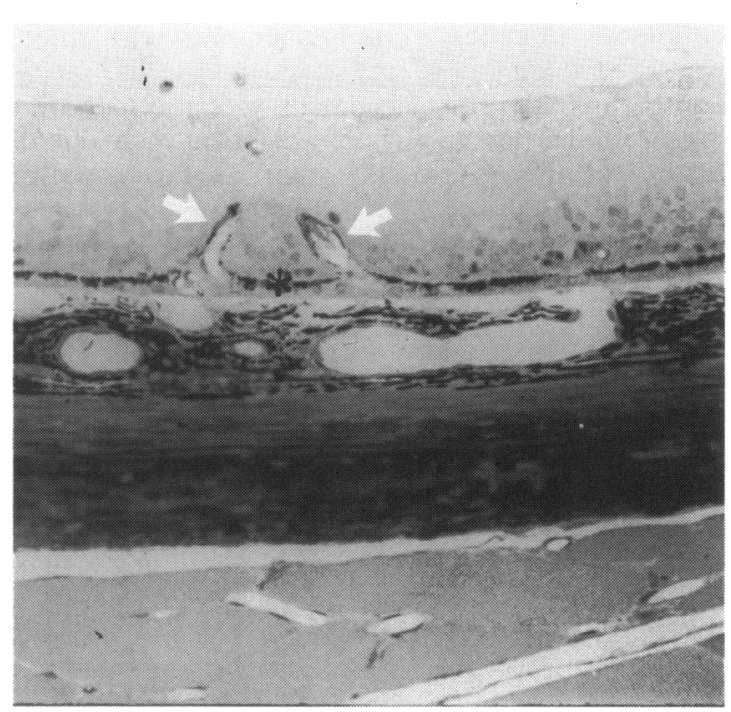

Fig. 2a Micrograph showing typical characteristics of urethane-induced retinopathy. Note loss of outer nuclear and photoreceptor layers; and presence of retinal vessels (arrows) extending into the retinal pigment epithelial layer $\left({ }^{*}\right)$. (Toluidine blue, $\times 180$ ).

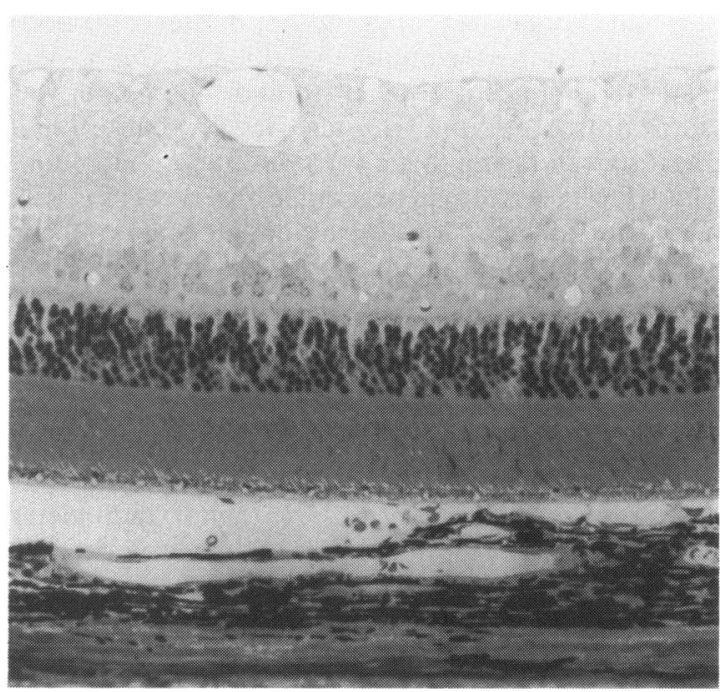

Fig. 2b Micrograph of normal control retina. (Toluidine blue, $\times 180$ ).

animals 14-16 weeks old. Thus comparison of VF values based on the duration rather than the severity of the retinopathy was not meaningful.

Fluorescence microscopy (Table 1) showed that the control animals (group A) had no abnormal permeability of BRB. In the retinopathic rats, however, there were instances in which no leakage of $\mathrm{NaFl}$ occurred into the neural retina even if some retinal 


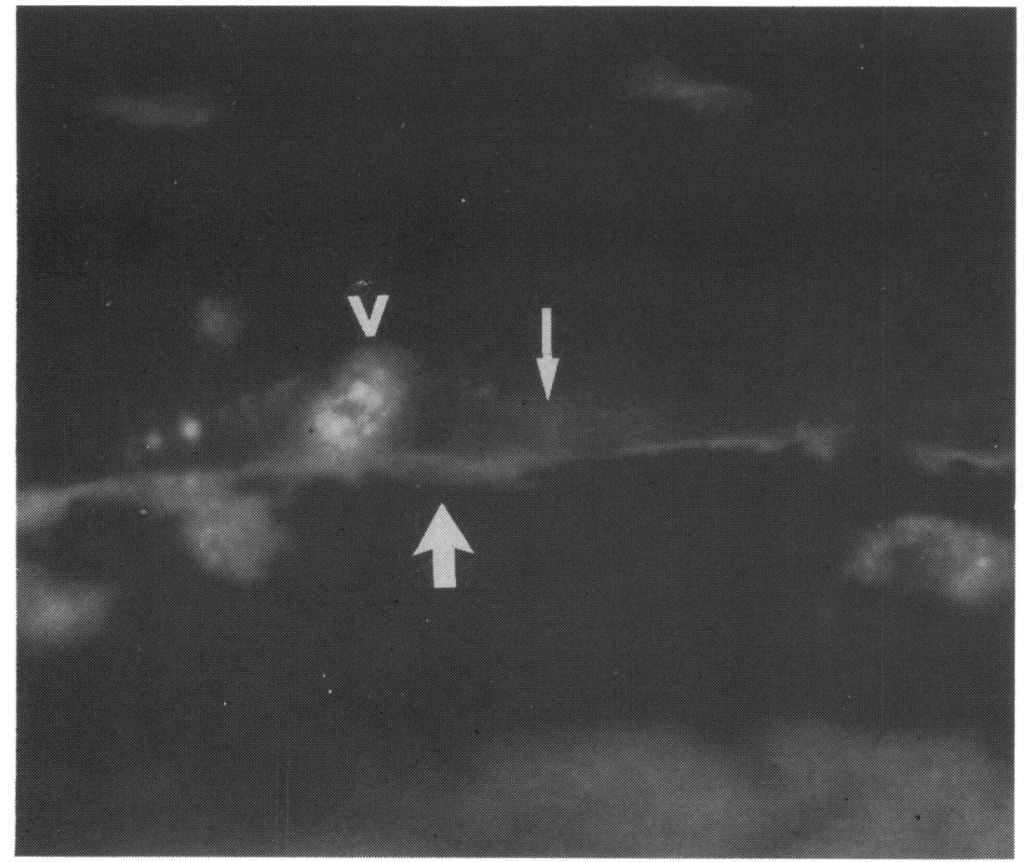

Fig. 3a Fluorescence micrograph showing vessel $(V)$ within the RPE layer (thin arrow) with fluorescence adjacent to the vessel. Note presence of choriocapillaris fluorescence (broad arrow) just beneath the RPE. $(\times 800)$.

vessels were present within the RPE (group B) and instances in which the intra-RPE vessels leaked $\mathrm{NaFl}$ (Fig. $3 \mathrm{~A}$ ) into the adjacent neural retinal (group C). In both groups $B$ and $C$, leakage from intra-RPE retinal vessels into the RPE layer was observed (Fig. 3B).
Vitreous fluorophotometric results expressed as mean vitreous:plasma ratios (V:P) are summarised in Table 1. Statistical analysis (Table 2) showed that the $\mathrm{VF}$ technique could distinguish between the control rats (group A) and the retinopathic rats (groups B

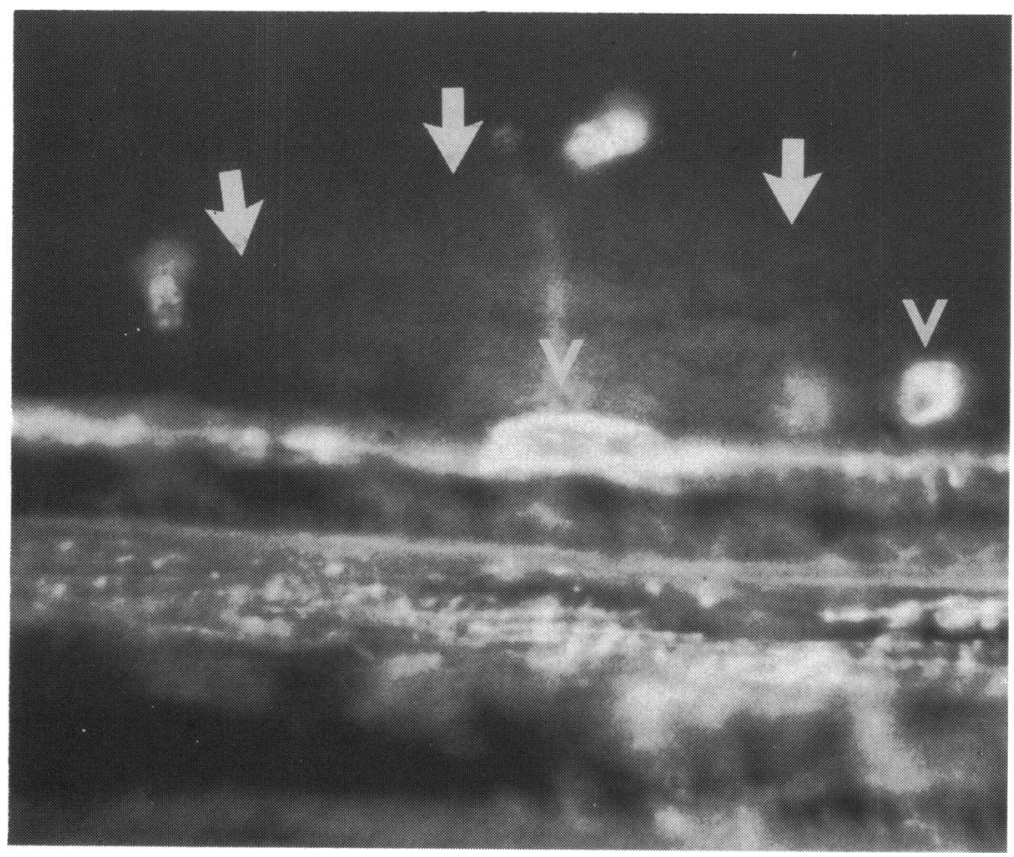

Fig. 3b Fluorescence micrograph showing vessels $(V)$ within the RPE layer and fluorescence spreading from that site into the adjacent neural retina (arrows). $(\times 800)$. 
Table 2 Analysis of $V: P$ data

\begin{tabular}{ll}
\hline Animal groups & Difference \\
\hline Control A vs. test B & Nonsignificant (NS) \\
Control A vs. test C & Significant $(p<0 \cdot 001)$ \\
Test B vs. test C & Significant $(p<0 \cdot 01)$ \\
Control A vs. test B and C & Significant $(p<0 \cdot 02)$ \\
\hline
\end{tabular}

$\mathrm{p}=$ Probability with Student's $t$ test.

and $\mathrm{C}$ ) as a whole at a level of confidence of $\mathrm{p}<0.02$. Further analysis based on the FM data showed that the nonleaky retinopathic rats (group B) could not be distinguished from the controls. However, the intraretinal leakage group $(C)$ could be distinguished from the control rats (A) and the nonleaky retinopathic rats (B) at levels of confidence of $p<0.001$ and $\mathrm{p}<0.01$ respectively.

\section{Discussion}

Fundus fluorescein angiography and vitreous fluorophotometry are 2 techniques wherein $\mathrm{NaFl}$ is used to assess permeability of the BRB in vascular retinopathies in man; these techniques have also been employed in the study of experimental rat retinopathies. In our present experimental study we could further define the permeability abnormality by identifying involved tissues by means of fluorescence microscopic techniques. This further definition, in turn, provided a more meaningful basis on which to assess the vitreous fluorophotometric data, in that the most significantly raised VF values were obtained in those rats (group $\mathrm{C}$ ) in which leakage of $\mathrm{NaFl}$ from retinal vessels into the neural retina was demonstrated by FM. Since a previous study ${ }^{6}$ had shown the presence of fenestrations in endothelial cells of the retinal vessels within the RPE, it is not surprising that leakage could be demonstrated histologically. However, in some rats with retinal vessels present within the RPE, leakage was confirmed to the RPE layer. This suggests that, even though the intrapigment epithelial retinal vessel is leaky (and presumably fenestrated), the barrier function of the RPE is intact and, in essence, continuing to function as an RPE/choriocapillaris unit.

The presence of $\mathrm{NaFl}$ within the neural retina of other retinopathic rats might suggest that (1) the retinal vessels are leaking from portions not within the RPE or (2) the RPE has also lost its barrier function. This present study does not support the first hypothesis in that retinal vessels outside the RPE were not noted by FM to be leaky. The second hypothesis is at present under investigation with electron microscopic permeability assessment techniques utilising electron-dense tracers to define the possible permeability abnormality of the RPE associated with the retinal vascular abnormality.

This complex relationship between the retinal pigment epithelium and vessels within the RPE tissue provides an opportunity to begin to understand the relationship of these same tissues in retinopathies of man such as senile macular degeneration and others characterised by subretinal neovascularisation. ${ }^{1314}$ And finally this study emphasises the unique opportunity provided by the several fluorescent marker techniques to investigate permeability abnormalities of the blood-ocular barriers. This is important in that vitreous fluorophotometry does not readily show if abnormal values are the result of increased permeability of the BRB and/or the blood-aqueous barrier. Increased ocular fluorophotometric values for aqueous humour have been reported in experimentally hypertensive rats ${ }^{11}$ and in young diabetic patients. $^{15}$

We thank Noel Roa, Mary Ellen Murphy, Judith. Channer, and Benny Day for technical and Patricia McMullan for secretarial assistance.

This study was supported by the British Diabetic Association (E.E.K.), by USPHS grant EY 02038 (R.W.B.), and by an unrestricted grant from Research to Prevent Blindness Inc.

\section{References}

1 Novotny HR, Alvis DL. A method of photographing fluorescence in circulating blood in the human retina. Circulation $1961 ; 24: 82-6$.

2 Cunha-Vaz J, de Abreu JRF, Campos AJ, Figo GM. Early breakdown of the blood-retinal barrier in diabetes. $\mathrm{Br} J$ Ophthalmol 1975; 59: 649-56.

3 Grayson MC, Laties AM. Ocular localization of sodium fluorescein. Arch Ophthalmol 1971; 85: 600-4.

4 Kirber WM, Nichols CW, Grimes PA, Winegrad A, Laties AM. A permeability defect of the retinal pigment epithelium: occurrence in early streptozocin diabetes. Arch Ophthalmol 1980; 98: 725-8.

5 Bellhorn RW. Permeability of blood-ocular barriers of neonatal and adult cat to sodium fluorescein. Invest Ophthalmol Visual Sci 1980; 19: 870-7.

6 Bellhorn RW, Bellhorn MS, Friedman AH, Henkind P. Urethane induced retinopathy in pigmented rats. Invest Ophthalmol Visual Sci 1973; 12: 65-76.

7 Rabkin M, Bellhorn MS, Bellhorn RW. Selected molecular weight dextrans for in vivo permeability studies of rat retinal vascular disease. Exp Eye Res 1977; 24: 607-12.

8 Waltman S, Krupin T, Hanish S, Oestrich C, Becker B. Alterations of the blood-retinal barrier in experimental diabetes mellitus. Arch Ophthalmol 1978; 96: 878-9.

9 Jones C, Cunha-Vaz J, Zweig K, Stein M. Kinetic vitreous fluorophotometry in experimental diabetes. Arch Ophthalmol 1979; 97: 1941-3.

10 Klein R, Wallow I, Ernest JT. Fluorophotometry 111. Streptozotocin-treated rats and rats with pancreatectomy. Arch Ophthalmol 1980; 98: 2235-7.

11 Dutton J, Krupin T, Waltman S, Kolons B, Becker B. Fluorophotometry in the blood-ocular barrier in experimental systemic hypertension. Arch Ophthalmol 1980; 98: 731-3.

12 Bellhorn RW, Bellhorn MS, Poll DS. Permeability of fluorescein- 
labelled dextrans in fundus fluorescein angiography of rats and birds. Exp Eve Res 1977; 24: 595-605.

13 Grindle CFJ, Marshall J. Ageing changes in Bruch's membrane and their functional implications. Trans Ophthalmol Soc UK 1978; 98: $172-5$.

14 Sarks SH, Van Driel D, Maxwell L, Killingsworth M. Softening of drusen and subretinal neovascularization. Trans Ophthalmol Soc UK 1980; 100: 414-22.

15 Waltman SR, Oestrich C, Krupin T, et al. Quantitative vitreous fluorophotometry: a sensitive technique for measuring early breakdown of the blood-retinal barrier in young diabetics. Diabetes 1978; 27: 85-7. 